LBL- -25371

DE89 006663

\title{
Nuclear Dissipation and the Order to Chaos Transition
}

\author{
W.J. Swiatecki \\ Nuclear Science Division \\ Lawrence Berkeley Laboratory \\ 1 Cyclotron Road \\ Berkeley, California 94720 \\ USA
}

June 1988

DISCLAIMER

This report was prepared as an account of work sponsored by an agency of the United States Government. Neither the United States Government nor any agency thereof, not any of their employees, makes any warranty, express or implied, of assumes any legal liability or responsibility for the accuracy, completeness, or uselulness of any information, apparatus, product, or process disclosed, or represents that its use would not iniringe privately owned rights. Reference herein 10 any specific commercial product. process, or service by trade name, tradematk, manulacturer, of atherwise does not necessatily constitute or imply its endorsement, recom* mendation, or favoring by the United States Government or any agency thereof. The views and opinions of authors expressed herein do not necessarily stute of reflect those of the United Siates Government or any agency thereof.

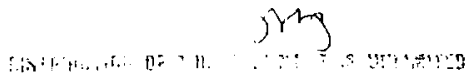


NUCLEAR DISSIPATION AND THE ORDER TO CHAOS TRANSIIION

\author{
W.J. SWIATECKI \\ Nuclear Science Division, Lawrence Berkeley Laboratory, University of \\ California, Berkeley, California 94720
}

The transition from ordered to chaotic nucleonic motions in the nuclear mean-field potential is reflected in the disappearance of shell effects in nuclear masses and deformations, and in the transition from an elastic, through an elastoplastic, to a dissipative behavior of the nucleus.

One of the major themes of contemporary science is the study of order to chaos transitions in dynamical systems. An abstract dynamical system is one whose $n$ degrees of freedom evolve in time according to certain specified mathematical rules. Remarkable progress in this general field is currently taking place. It is described in the following way in a book on order and Chaos by Bergé, Pomeau and Vidal:" "In recent years a new kind of physics has been emerging, which has been variously termed nonlinear, turbulent or chaotic. Its extremely diverse subject matter includes hydrodynamical turbulence, chemical kinetics and the study of electronic circuits. The similarity in the structure of these diverse phenomena results from a profound mathematical theory: the modern theory of nonlinear systems... The field of investigation in such studies afrars quite large, since it encompasses the analysis of all time-dependent phenomena. Treating the major types of behavior or evolution without direct reference to the actual matter through which they are manifested, this body of doctrine resembles a physical theory as powerful and structured as, for example, thermodynamics."

A sub-class of such theories especially relevant to the nuclear problem deals with conservative systems where the time development is formulated in terms of a Hamiltonian function of the $n$ degrees of freedon and the $i r$ conjugate momenta. The corresponding $2 n$-dimensional phase space is the natural arena in which to describe the evolution of the system. The classification of various structures that appear in this space for generic types of Hamiltonians has been the subject of intense research in the recent past. The explosive growth of this field is associated with the discovery of an unsuspected gold mine in this ancient discipline of analytical dynamics. The gold mine is located in what used to be thought of as an uninteresting. arid desert between two well-cultivated coastal regimes, the regime of 
integrable systems and the regime of chaotic systems. The term 'integrable system' refers to a rather special limiting situation, where there are as many constants of motion as there are degrees of freedom. In this case the evolution of a system with given initial conditions explores only a fraction of the available phase space. For a bound system this is usually in the form of an n-dimensional torus. Different initial conditions define different tori. In the fully chaotic regime a system started with even a single initial condition explores all of the phase space that is not excluded by a few overall conservation laws. This is the regime of Boltzmann's statistical mechanics, with a probability fog permeating phase space in place of the tori. Until relatively recently it was generally belfeved that the intermediate regime between the integrable and the chaotic limits is a dull area where tori dissolve gradually into the Boltzmann fog. What was found instead is that the intermediate regime has a wonderful structure, with necklaces of tori within tori within tori in a fractal succession. interspersed with chaotic regimes of fog, and with webs of interconnections almost erganic in appearance. Instead of a desert, there is a luxuriant rain forest. which changes in intricate ways as the deviation from integrability of the Hamiltonian is cranked up. The discovery of this gold mine in theoretical dynamics may rank in importance with the discoveries made by Hamilton and Boltzmann in the ordered and chaotic regimes. The new structures uncovered are in fact much richer than those in the familiar regimes. Fig.l is an attempt to give an impression of this modern view of dynamics. On the left
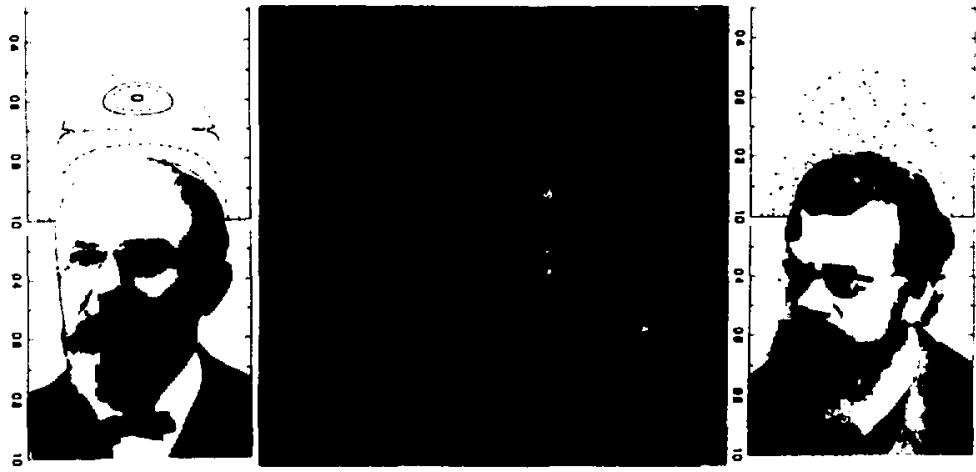

FIGURE 1

Pictures of phase space: Poincare tori on the left, Boltzmann fog on the right and the fractal jewels of the generic Hamiltonian phase space, as illustrated by the 'circle map' from ref. 2 . 
you see Poincare crowned with his tori. On the right sits Baltzmann wreathed in fog. The central panel, taken from ref. 2, gives an impression of what the real generic phase space looks like. I cannot resist quoting Michael Berry's rapturous description of this new landscape. In commenting on a sketch of such a phase space in his 1978 article on "Regular and lrregular Mation" ${ }^{3}$ he says: "I do not know who first drew this astonishing picture; but even the detail shown is a woefully inadequate approximation to the true situation. What a wonderful hierarchy! Near each rational invariant curve there are hyperbolic fixed points with associated chaotically wandering curves, and elliptic fixed points surrounded with invariant curves which repeat the whole structure ad infinitum...a lacework of intimate intermixing of integrable and stochastic motions. It must be emphasized that all this is in no sense pathological. It is the generic situation for solutions of Hamilton's equations."

What has all this to do with nuclear physics? The explosive growth in order-to-chaos research is due in large measure to computer studies of relatively simple, nonlinear, non-integrable systems, such as the Henon-Heiles two-dimensional anharmonic oscillator, ${ }^{4}$ or the stadium potential. ${ }^{5}$ (The former refers to the motion of a mass point in a potential well of finite depth whose equation in polar coordinates is $v=(1 / 2) r^{2}-b r^{3} \cos 30$, the latter to an oval cavity with impenetrable walls.) Even such simple systems exhibit many of the fascinating features of the generic order-to chaos transition but, despite great efforts expended on them in the past decade. unexpected discoveries continue to be made, and several questions remain unanswered, especially in the quantal versioll of the order-to-chaos problem. But the relevance of these studies to nuclear physics, and the relevance of nuclear physics to order-to-chaos research, is at least obvious. A good starting point for nuclear theory is the mean-field approximation, in which nearly independent nucleons move in a comfion potential well. This potential well does in fact resemble an anharmonic oscillator for light nuclei and a cavity for heavy nuclei. Thus, much of nuclear theory done in the past has been unwittingly exploring aspects of the order-to-chuos problem, in fact the especially interesting quantal version of the problem. However, with one notable exception. the insights gained in nuclear physics have not been incorporated in order-to-chaos research. (The exception is the random matrix theory of nuclear level spacings and statistical reaction theory.) Conversely, it is only recently that nuclear physicists have become generally aware of order-to chaos research that is relevant to nuclear physics.

In order to illustrate the similarities in the two originally independent 
branches of science, fig. 2 compares the phase space of the classic Henon-Heiles problem, and of planar particle trajectories in an idealized spheroidal nuclear potential. ${ }^{6}$ In both cases one has a two-dimensional potential well of finite depth, which may be described by a simple function of two cartesian coordinates $x, y$. In the nuclear case the well is like a Woods-Saxon potential and the problem is integrable if the well is spherical but becomes non-integrable for a deformed nucleus. The same is true for the

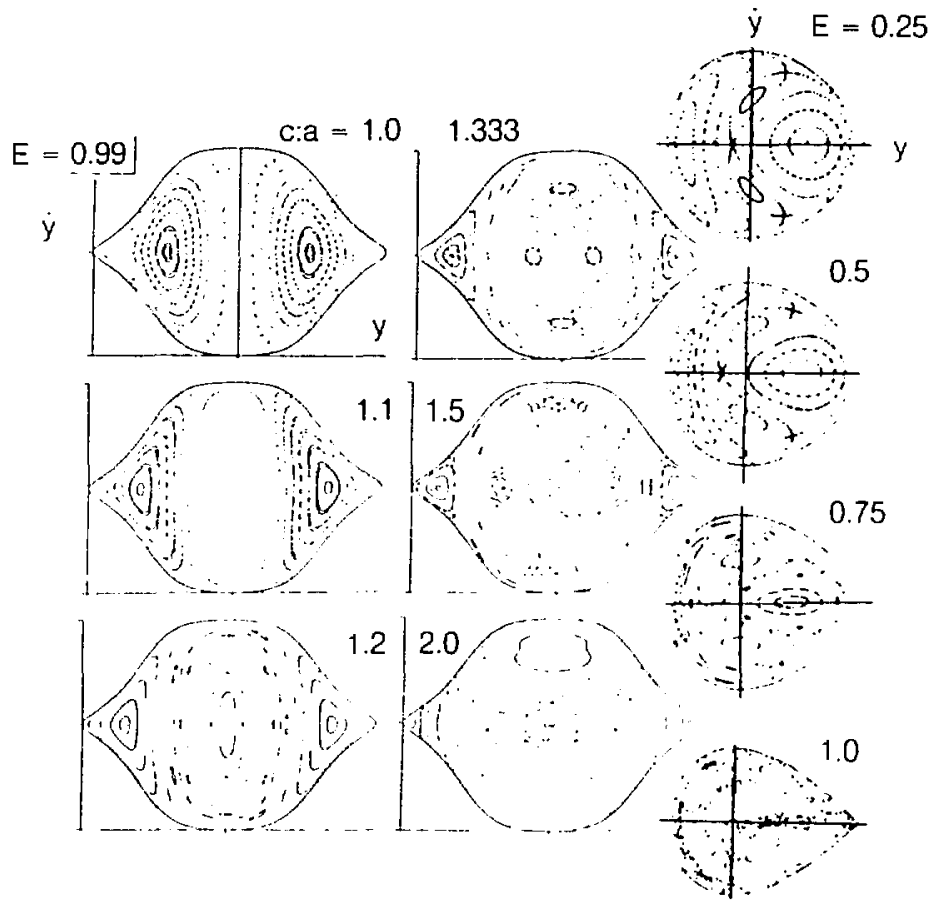

×BL 8858439

FIGURE 2

Poincaré sections for the Hénon-liejles problem at energies from $25 \% 10100 \%$ of the escape energy (right) 4 , and for a particle with $99 \%$ of the escape energy moving in a diffuse potential representing a light nucleus with axes ratios increasing from 1 to 2 (left) 6 . 
Henon-Heiles potential, where integrability is lost as the particle energy is raised from small values towards the escape energy. What is shown in fig. 2 are Poincaré sections through the phase space, the sections being by the $y, \dot{y}$ plane at fixed $x$ (the value of $\dot{x}$ follows from energy conservation). Consecutive intersections of this plane by a particle trajectory with a given initial condition form a series of dots. The dots lie on a closed curve if the motion in phase space is on a Poincare torus. If the motion is chaolic the dots generate a Boltzmann fog. The left part of fig. 2 shows how the transition from order to chaos takes place as a function of increasing deformation in the nuclear case. The right hand portion shows a simflar sequence for the much studied Henon-Heiles problem. The theory of the generic pattern sequences in such a transition from order to chaos has been the subject of intense research in analytical dynamics.

A second example of investigations of similar problems in nuclear physics and in order-to-chaos dynamics is shown in $\mathrm{fig}$. 3 . The upper part is taken from an exhaustive recent study entilled "The quest for quantum chaos",

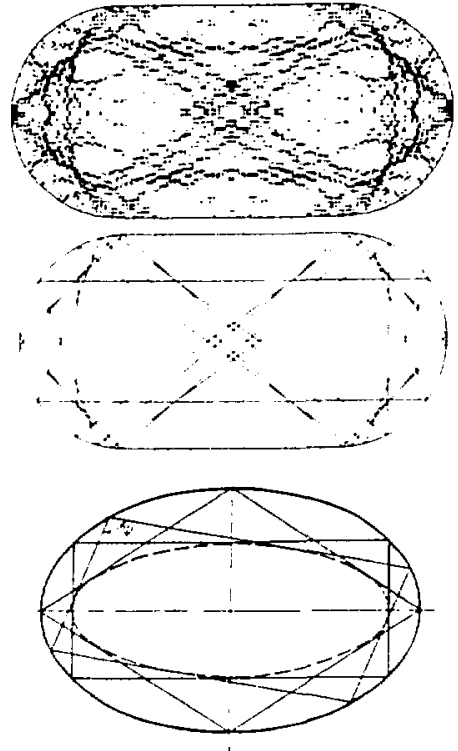

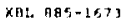

FIGURE 3

The intensity pattern of a very high eigenmode in the stadium potential (top). 5 Regular patterns like these are the rule, although the classical orbits that seem related (middle part) are nighly unstable. Studies of planar orbits in the integrable spheriodal cavity (bottors) are important in understanding nuclear deformations.? 
attempting to relate classical and quantal trajectories in the stadium potential. In this remarkable work, carried to the limits of available computer power, the eigenfunctions of the wave equation can be solved numerically for thousands of eigenstates. Dne of the unexpected results was the ubiquity of wave functions with eastly recognizable similaritirs to highly unstable classical trajectories. The classical stadium problem is one which, in a technical sense, has been proved to be chaotic because of such instabilities. Yet, in the quantized version, patterns of trajectories that one might have thought would be highly evanescent, show up all the time. The suggestion has been made that this might be due to the relative proximity of the stadium to the spheroidal shape, which corresponds to an integrable problem (for any eccentricity). The bottom part of fig. 3 is taken fron a 1977 study, ${ }^{7}$ in the nuclear context, of classical and semiclassical trajectories in such a spheroidal cavity. The aim was to achieve a simple understanding of nuclear deformations. The two problems, quantum chaos in the stadium and shell effects in deformed nuclei, are clearly related, but I believe these relations remain largely unexplored.

What are some of the general lessons we have learned about the nuclear problem that we now realize are governed by the order-to-chaos transition in nucleonic motions? In the case of static nuclear properties, the outstanding qualitative lesson could be stated like this:

*** When the nucleonic motions inside the nucleus are integrable, one expects to see strong shell effects in nuclear structure, e.g. in nuclear masses and deformations.

*** When nucleonic motions are chaotic, one expects smooth, statistical, Thomas-Fermi, Oroplet Model approaches to be good approximations. This is because phase space is much more uniformly filled with the Boltzmann fog in this case.

Before going on to the question of nuclear dynamics and dissipation let me remind you of some of the simplest unanswered questions in the realm of statics. One problem is to understand the washing out of shell effects. Thus. the macroscopic-microscopic approach to nuclear energies, in particular the Strutinsky shell correction method, is based on synthesizing the insights obtained in the two limiting regimes of integrable and chaotic systems. But 1 believe it is true to say that a proper understanding of the intermediate regime is still lacking. How do shells dissolve with deviations from integrability or, conversely, how do incipient shell effects emerge as the system first begins to feel its proximity to an integrable situation? This 
question has, for a good reason, remained largely unanswered: even at the classical level, and even for simple schematic problems like the stadium, the iransition from of order to chaos exhibits unexpected and intricate features. For example. it was only the discovery of the famous Kolmogorov-Arnold-Hoser theorem in analytical dynamics that revealed how a generic integrable system has the amazing tendency to hang on to its integrable-like behavior in the face of non-integrable perturbations. The KAM theorem states, in effect, that "tori are tough." Fig. 4 illustrates this point symbolically. (It also notes

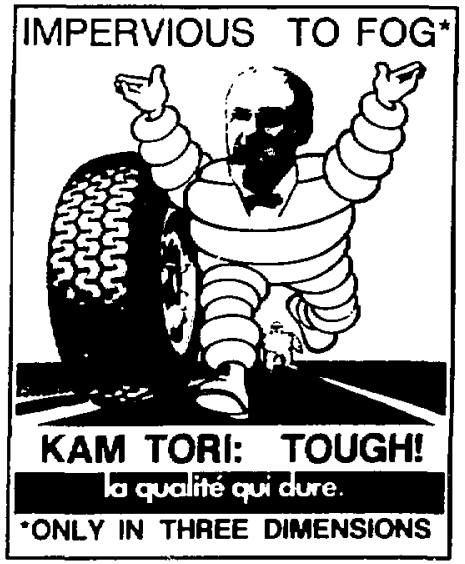

FIGURE 4

The Kolmogorov-Arnold-Hoser theorem demonstrated the enduring character of tori. Hence their importance as an organizing element for the phase space of non-integrable systems, impeding the spread of chaos.

the important fact that in 3 dimensions tori serve as barriers preventing the spread of Boltzmann fog. i.e.. of chaos, from one region of phase space to another. In more than three dimensions the fog can sneak around the tori in what is called Arnold diffusion, a subject of active current research.)

The relevance of the toughness of KAH tori to nuclear physics may be at a quite fundamental level. Thus, the relative accuracy of the nuclear independent-particle model may be due nut only to the Paulj priniciple. which suppresses two-body correlations between nucleans, but also to the KAM theorem which helps with the survival of integrable-like. independent-particle properties in the face of residual nucleon-nucleon interactions. More generally, the usefulness of a quasi-particle approach in the theory of fermi liquids may be related to the toughness of KAM Lori. In a way, we owe a debt of gratitude to Kolmogorov, Arnold and Hoser for the applicability of strutinsky's shell correction method to nuclei. But it also follows that to 
understand how shell effects are washed out when the nuclear shape moves away from integrable configurations, or when the nuclear temperature is increased and the Pauli blocking becomes less effective in suppressing the nonintegrable features of the residual interactions, we will need help from general theories of the transition from order to chaos in quantal systems.

The type of problem one is dealing with here is illustrated in the schematic example in fig. 5, which shows the total energy of A quantized particles of mass in in a spherical cavity with radius $r_{0}{ }^{1 / 3}$. If the

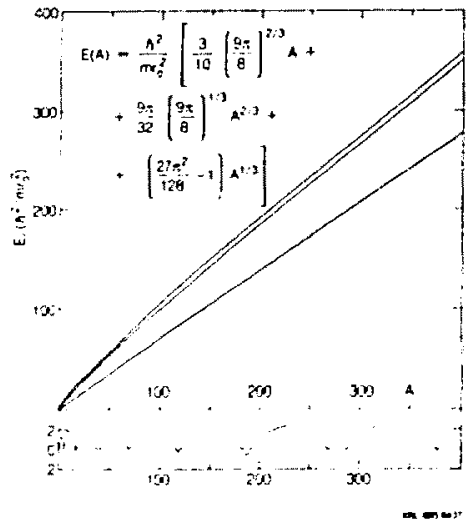

\section{FIGURE 5}

The energy of A particles (4 per eigenvalue) in a spherical cavity of radius $R=r_{0} A^{1 / 3}$. The smooth curves are successive statistical approxinations, to order $A, A^{2} / 3$. $A^{1 / 1}$; appropriate in the chaotic limit. The curve at the bottom represents deviations of the exact result from the highest-order approximation. These deviations are associated with the presence of tori in phase space caused by integrability.

phase space could be considered as filled with Boltzmann fog. one could apply ine following asymptotic formula (due to Weyl-Balian-Bloch-Baltes') for the average density of eigenvalues, $\rho(k)$. per unit interval of wave number, $k$. in the case of a cavity with volume $V$, surface area $S$ and integrated curvature $k$ (given by the surface integral of the local curvature $R_{1}^{-1}+R_{2}^{-1}$ ):

$$
\rho(k)=V k^{2} / 2 \tau-S k / \theta \tau+k / 12 \tau^{2}+\ldots .
$$

For a sphere this leads to the following expression for the total energy uf A particles:

$$
E(\Lambda)=\frac{n^{2}}{m r_{0}^{2}}\left[\frac{3}{10}\left(\frac{9 \pi}{\theta}\right)^{3 / 3} A+\frac{9}{32}\left(\frac{9 \pi}{\theta}\right)^{2 / 3} A^{3 / 3}+\left(\frac{2}{12} \frac{y^{2}-1}{28} A^{2 / 3}+c+\ldots\right],\right.
$$

where $c$ is a constane, not jet calculated andlytically, but equal 20 about $i$ according to fig. 5. Ihis figure shows the prediction of eq.(2) to order $A$. 
$A^{2 / 3} \cdot A^{1 / 3}$, as well as the remaining deviations. These deviations are, like a Sorutinsky shell correction, the differences between a sum over eigenvalies and a smooth approximation. Now suppose the sphere is distorted and the potential well is made diffuse, or residual interactions are switched on. How will the shell effect fluctuations decrease with increasing size of such non-integrable perturbations? We now realize that this is a question concerning the transition from order to chaos, i.e. the destruction of Poincaré tori in phase space. To understand properly this transition we need the help of the experts in this field, often outside nuclear physics, who have concerned themselves with this general problem for some time.

A simpler question along these lines, which also has not been answered, concerns the completely chaotic limit. Suppose the shape of the potential in which the non-interacting particles are contained has lost all integrability, and the Boltzmann fog has taken over. Assume that we are now allowed to use the well-established random matrix theory to discuss the distribution of the eigenvalues in this potential. ${ }^{9}$ (The Weyl-Balian-Bloch-Baltes formula gives the average level density and the random matrix theory provides information about fluctuations around this average.) Suppose one now sums the fluctuating eigenvalues and subtracts a smooth average. How big will the remaining deviations be? I am not aware of a formula for. say. the RMS value of these residual deviations, but such a formula is surely just around the corner. since all the necessary information seems contained in the random matrix theory. 10 A formula like this would be a valuable addition to the macroscopic-microscopic theory of nuclear masses and deformations, as it would represent a certain irreducible limit on the accuracy of Liquid-Drop or Thomas-Fermi type approximations. Will this limit come out to be $1 \mathrm{HeV}$ or $0.1 \mathrm{HeV}$ for a typical nucleus? State-of-the art macroscopic-microscopic mass formulae are now accurate to 1 MeV or less, and it would be nice to know at what level the fluctuations in the density of the Boltzmann fog become relevant.

Now to the question of dynamics. How does an idealized system composed of nearly independent particles in a potential well respond to the potential's time dependence, for example to changes in shape? Huch less is known in a rigorous way about this question. considered as an abstract dynamical problem. This is true eyen at the classical level and our ignorance is worse in the quantal ca:-4. In the special context of nuclear physics, a large body of valuable experience has, indeed, been accumulated, usually without the realization that the problem is intimately related to the transition from 
order to chaos in nucleunic motions. The general lesson emerging from such studies of nuclear dynamics seems to be along the folluwing lines"

$\star \star \star *$ When the nucleonic motions inside the rucleus are integrabla, the response of the nucleus to shape changes should resemble that of an elastic solid.

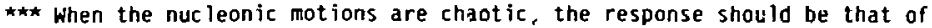
a very viscous fluid, with an unusual type of viscosity.

*** In the intermediate regime an elastoplastic behavior is exfected.

In a qualitative way these expectations go back many years, to $\mathrm{Hi}$ il and whee ler ${ }^{12}$ and to Süssmann, 13 among others. They are based on the observation that for an integrable systim the energy spectrum will be qualitatively similar to that of a Hill-wheeler box or, to take a somewhat more realistic siape, to a spheroidal cavity. 14 Fig.6 shows the energy spectrum of an N-particle system in sush cavities. Each parabola-like curve corresponds to deforming the system with the particles hanging on to their constants of the motion, i.e. to their quantum numbers. In the example of the box (but not for the spheroid) this corresponds simply to stretching each wave function along one axis in a volume-preserving way. One readily verifies the following theorem: a system of any number of independent quantized particles, in a potential of any form, whose wave functions are stretched in a volume-preserving (quadrupole) way, responds near an equflibrium configuration with a stiffness that is twice its kiretic energy conteni. Thus, if we write the increase of the kinetic energy $E$ as

$$
\Delta E=(1 / 2) K a^{2} .
$$

where $\&$ specifies the relative increase of the dimension of the axis being stretched, then the stiffness $K$ is given by $2 E$. For a Fermi gas of $A$ particles with Fermi energy mo $v^{2} / 2$ and energy per particle three fifths of the Fermi energy. this gives

$$
K=2(3 / 5)\left(m v^{2} / 2\right) A=(3 / 5) M v^{2}
$$

where $M$ is the total mass of the system. Thus the stiffness is proporticnal to the total mass or tctal particle number and is, therefore, a bulk property, like the stiffness of $\Rightarrow$ solid. Incidentally. the inertial resistance of a spherical mass $M$ with radius $R$ against volume-preserving quadrupole stretchings is given by

$$
H_{\text {eff }}=(3 / 10) M R^{2} \text {. }
$$


so that the frequency of the associated giant quadrupole resonance would be

$$
\omega=\sqrt{K / M_{e f f}}=\sqrt{2} \mathrm{~V} / \mathrm{R}
$$

This turns out to be in remarkably close agreement with measurements. "I

Coming back to fig. 6, if during the deformation of the system the particles can be induced to give up their constants of the motion and to redistribute themselves into the lowest energy states at each deformation, then the stiffness of the system will correspond to the much softer envelape of the parabnla-like curves. One can readily verify that for a system with a
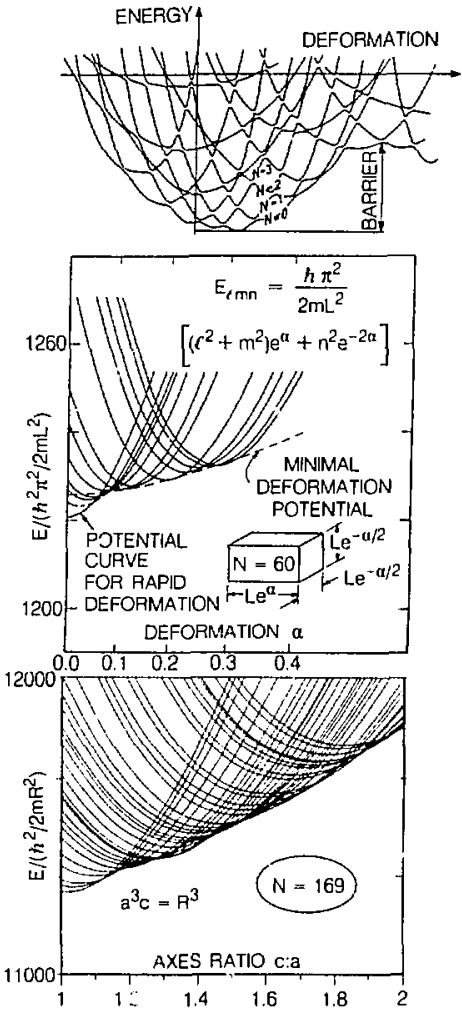

XEt Bas 64A
FIGURE 6

In an integrable system corresponding to $\mathrm{N}$ particles in a $\mathrm{Hill}-$ thee ler box (middle pane $I$ ) or a spheroidal cavity (bottom panel), the deformation-energy spectrum consists of parabola-like curves characterized by a solid-like stiffness. The envelope of these curves defines a softer, fluidlike deformability. As integrability is destroyed, most energy levels begin to avoid each other (top pane1), and an elasto-

plastic behavior is expected. 
thin surface, such as a classical or quantized gas in a cavity, the stiffness of this envelope is proporticnal to $A^{2 / 3}$ (or, for a fixed $A$, to the surface area of the system) so that one is now dealing with the stiffness of a fluid endowed with a surface tension. For a Hill-Wheeler box these features had been studied exhaustively by, among others, C.F. Tsang, from whose 1969 thesis ${ }^{15}$ fig. 7 is adapted. The top parts of the figure were drawn to bring out the similarity with fig. 5. The bottom part verifies the predicted dependence of the envelope on deformation. But what does it take to make the particles give up their constants of the motion and redistribute themselves into the most comfortable energy states defining this envelope? Evidently one must perturb the integrability of the system sufficiently so that the constants of the motion, i.e. the tori in phase space, i.e., the quantum numbers in the quantized problem, i.e. the parabola-like curves in the energy spectrum, lose their significance. If these features disappear entirely, one is in the chaotic regime and the fluid-like behavior should be in evidence.
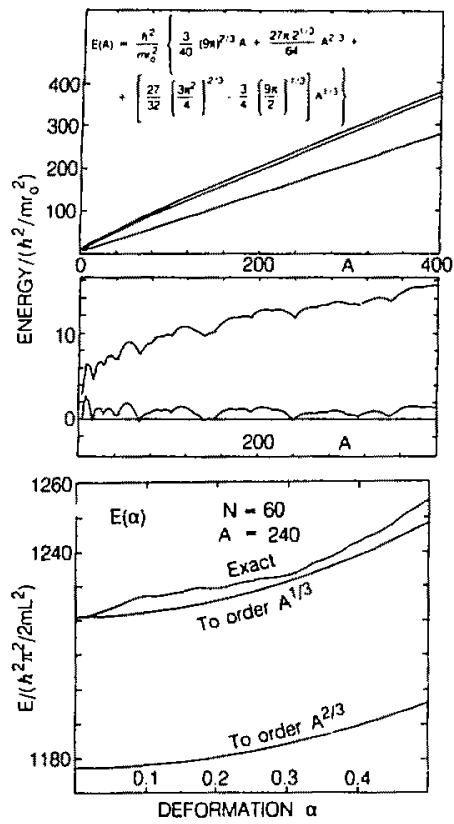

xax as 0442

FIGURE 7

The top panel is like Fig. 5, but for the Hill-whee ler box. The middle panel displays the exact energy after subtraction of the statistical approximations (to order $A^{2 / 3}$ and $A^{1 / 3}$ ). The bottom panel verifies the theoretically calculated response of the system to stretching of one side of the box by expa with contractions of the other sides by $\exp (-\alpha / 2)$. 
What kind of fluid will it be as regards its dynamic behavior? There exist idealized studies $11,21,22$ of this 'chaotic regime' dynamics (questioned as regards their relevance to the nuclear problem in ref. 16) which suggest that the fluid should be very viscous, with a novel type of viscosity, the so-called 'one-body dissipation.' This dissipation is typified by the following 'wall formula' for the rate of energy flow from the shape degrees of freedom to the particles of the gas

$$
\frac{d E}{d t}=\rho \bar{v} \phi h^{2} d \sigma .
$$

where $\rho$ is the mass density of the gas, $\vec{v}$ is the mean particle speed, equal to $(3 / 4) v$ in the case of a degenerate Fermi gas, $n$ is the normal velocity of a surface element $d \sigma$, and the integral is cver the surface of the container, assumed in this case to be deforming without overall drifts or rotations.

In general, a nuclear system will be neither fully integrable nor fully chaotic. Its energy spectrum will look more like the schematic drawing in the upper part of $\mathrm{fig}$. 6, adapted from Süssmann. 13 The dynamical behavior of the system will now depend on the speed of the deformation. For slow deformations the system will follow the fluid-like lowest-energy curve. For fast deformations it will be inclined to jump across the gaps at the avoided ievel crossings, the more efficiently the higher the deformation cpeed. Thus. at a given instant. some of the energy will be stored as elastic energy, but some will be drained out in the form of dissipation as the particles rearrange themselves into more sensible patterns of motion appropriate to a given deformation. It is especially $w$. Nörenberg and his collaborators who have been developing theories of such elastoplastic behavior. 17 This scheme is epitomized by the introduction of a type of force (attributed to Maxwell) whose magnitude is not just proportional to the instantaneous velocity a of a coordinate $q$, as with ordinary dissipation, but to an exponentially weighted integral over the time history of this velocity:

$$
F=-c \int_{t_{0}}^{t} d t^{\prime} e^{-\left(t-t^{\prime}\right) / \tau} q\left(t^{\prime}\right) .
$$

Such a force has the property of acting in a dissipative way for slow deformations and in an elastic way for fast deformations.

The above theories are only two examples of studies of nuclear dynamics that bear to a greater or lesser extent on the question of nuclear dissipation. I should mention especially the transport type theories of 
Weidenmiiller and collaborators, the linear response theories typified by the work of Hoffmann, Siemens and others, the theories of nuclear collisions with friction due to Gross and collaborators, hydrodynamic studies of fission and nuclear collisions by Nix. Sierk and others, the master-equation treatment explored by Moretto and collaborators, and the particle-exchange theory of nuclear collisions due to Randrup. Feldmeier. Ddssing and others. Most prominent are, of course, the extensive simulations of nuclear dynamics based on the time-dependent Hartree-Fock method, initiated in the nuclear context by Kerman, Koonin, Negele, and extended by several groups. Extensive references to the above theories, as well as confrontations with experimental data can be found in refs. 19, 20, and 23. Here I would like to make just one commeit about all these theories, including those I mentioned earlier. They have one feature in common. namely that they were developed at a time when the relevance of the order-to-chaos transition in the nucleonic motions to the question of nuclear dynamics was not fully appreciated. Take as an example the TDHF theories. Because of the key role of integrability, it seems clear in retrospect that in order to have a chance of forming a reasonable estimate of a nuclear system's response to shape changes, whether alastic, elastoplastic or dissipative, one needs to pay particular attention to the breaking of symetries and to the inclusion of residual interactions. A theory with artificially imposed symetries and without residual interactions may be very adequate for static problems, but it cannot be expected to provide a good guide to dissipative effects.

Let me end by giving you a sample from a recent study concerned with illustrating the relation between the transition from order to chaos in the individual-particle motions of an idealized system to its dynamical response as a whole. 21 Figure 8 shows what happens to the energy content of a gas of non-interacting particles in a container, when the container is made to oscillate periodically around the spherical shape. In the top and middle parts of the figure the deviations of the surface of the container from the sphere are proportional to Legendre polynomials of order $2,3,4,5$ and 6 . In the bottom part the oscillations are exactly spheroidal, so that without the time dependence the problem of the particle motions would be exactly integrable. In all cases the time dependence is slow (adiabatic) with respect to all but the slowest particles of the gas. The different curves show the relative increase in the energy content of the gas for five complete periods of the oscillation:. (The motion is started from rest at the maximum amplitude of the deformation.) In the case of the distortions $P_{3}, P_{4}$. 


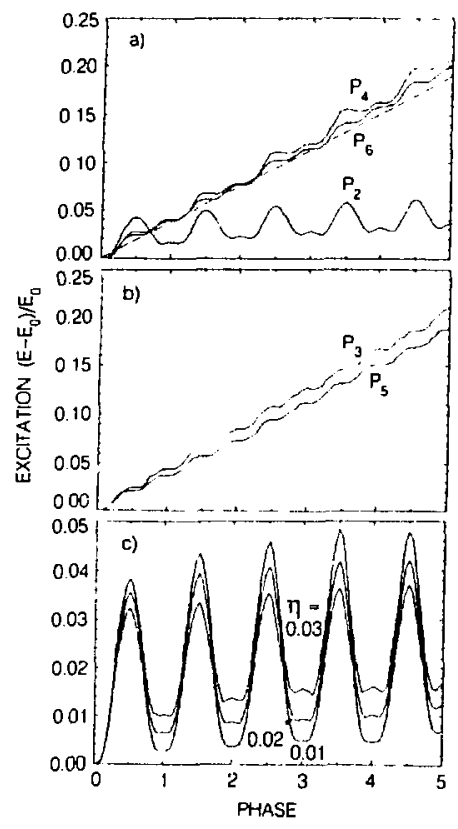

XBL $885 \cdot 8440$

\section{FIGURE 8}

The relative energy change of a gas of particles inside a cavity undergoing five complete oscillations around the sphere. In the bottom panel the oscillations are spheroidal and, with decreasing frequency (as labeled by $n$ ), the response tends to a reversible, elastic one. The index $n$ is the ratio of the maximum tip speed to the Fermi velocity $v$. The curves labeled $P_{n}$ refer to oscillations proportional to Legendre polynomials. Except for $P_{2}$ the resulting monotonic, dissipative energy increase is close to that predicted by the wall formula, whose overall trend is indicated by the dashed line.

$P_{5}, P_{6}$, the increase of the energy is monotonic, corresponding to a purely dissipative response of the system. This response is, in fact, in close agreement with the wall formula, eq.(5), whose prediction would be indistinguishable from the curve labeled $P_{5}$. The case of (small) $P_{2}$ deformations represents near-integrability, and the response of the ystem is partly elastic, as shown by the reversible bumps in the energy superimposed on a dissipative background. In the case of the spheroidal oscillations in the bottom part of $\mathrm{fig}$. $B$ the response is largely elastic, tending in fact to perfect reversibility as the speed of the oscillations. characterized by the adiabaticity index $\pi$, tends to zero. These different responses illustrated in fig. B, varying from dissipative to elastic, are brought into correspondence with the arder-to-chaos transition in fig. 9 . This figure shows samples of Poincaré sections of the phase space for particle trajectories in variously deformed static containers. In this sample all the 


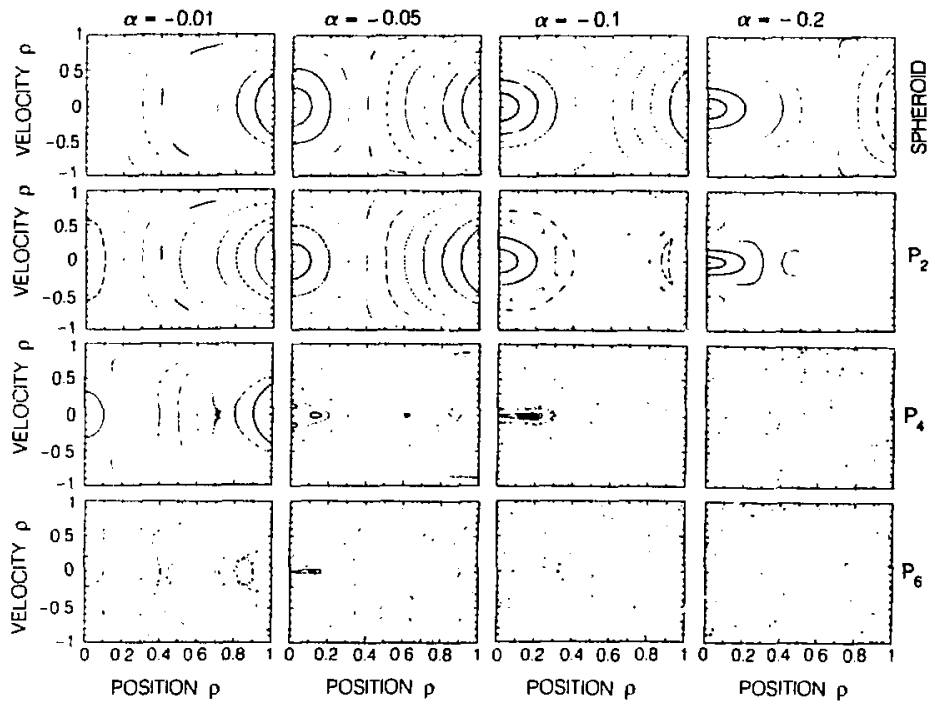

XEL $885 \cdot 8438$

\section{FIGURE 9}

Poincare sections by the plane containing the cylindrical polar coordinate $p$ and velocity $\dot{p}$ for a sample of 10 particles of the gas studied in $\mathrm{fig} .8$. Tori give place to chaos as the amplitude a of the non-integrable deforinations is increased. (Negative values of a correspond to oblate shapes.)

trajectories are in a plane containing the axis of symmetry. The magnitude of the deformation is given by the parameter $a$, which for the spheroid is the relative elongation of the major axis. The negative values of a indicate oblate deformations. For the polynomial types of distortion the amplitude is chosen so as to result in the same RMS deformation as for the spheroidal distortion at the given $a$. In the case of the spheroid the poincare sections at all deformations show the existence of tori, in agreement with expectation. In the case of $P_{4}$ and $P_{6}$ there is largely chaos, except for small deformations. (The survival of these tori for not too large non-integrabie perturbations is an illustration of the KAM theorem.) The case of $P_{2}$ deformations is intermediate: for small to maderate deformations the tori survive as for the spheroid, but eventually chaos begins to invade the 
phase space. Thus fig. 9 provides a qualitative explanation of the dynamic behavior seen in fig. B. It also provides a crude 1 llustration of the generic patterns of tori within tori, characteristic of the initial stages of the order-to-chaos transition. The full richness of these structures is not really in evidence because the plots in each panel of fig. 9 are based on only ten initial conditions for the particle trajectories, and much of the detailed structure is lost because of the cuarseness of this scan.

The theme of my talk was the relation of the order-to-chaos transition to nuclear dissipation. My emphasis has been on taking some of the familiar elements of nuclear physics and relating them to the expanding field of nonlinear dynamirs. Much remains to be learned about this relation even at the classical level, but the outstanding problem is, of course, to generalize the discussion to the quantal level. A conceptually simple study that suggests itself is a thorough investigation of a quantal gas of non-interacting particles in variously deforming time-dependent containers. Figure 10 illustrates one such study, now ten years old, and I chose it to stress both the paucity of such elementary calculations and the continued lack of understanding of the numerical results. (Why does the result for the

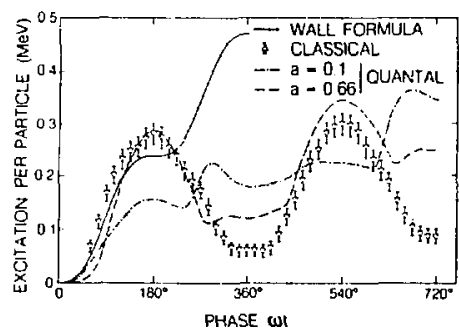

atersens

\section{Figure 10}

One of the few ayailable studies of a quantized gas of independent partic!es in an oscillating spheroidal potential, representing an idealized medium weight nucleus. 22 The classical calculations are for a container with zero surface diffuseress. The quantal calculations are for a realistic value of the diffuseness $(a=0.66)$ as well as for a much smaller one $(a=0.1)$. Why does the larger diffuseness give results more nearly like the classical (closely reversible) plot?

quantal problem in the more diffuse well resemble the classical calculation in a sharp we11?) One thing that we have learned is that there are good reasons why even such an idealized problem is full of subtleties. I believe that understanding thoroughly such model calculations should be helpful in making progress with the much more difficult realistic nuclear problem.

Let me finish with the following thought. The field of nuclear physics nas been revitalized recently by two major developments. One is the establishment of contacts with the subhadronic world of Quantum Chromodynamics. The other is the contact with the world of Quantum chaotic Dynamics. The former 
is the contact with the world of Quantum Chaotic Dynamics. The former establishes a bridge to the fundamental field of particle physics, the latter to the universal field of nonlinear dynamics. Working in nuclear physics is not a bad way to keep in touch with both.

\section{ACKNOWLEDGMENT}

I would like to thank J. Blocki and Y.-J. Shi for their part of the research reported in Figs, 8 and 9, and F. Brut for part of Fig, 6,1 am grateful to several colleagues for introducing me to the subject of chaos and nonlinear dynamics, among them R. Arvieu, O. Bohigas, F. Brut, A. Kaufmann and A. Lichtenberg. This work was supported by the Director, Office of Energy Research, Division of Nuclear Physics of the Office of High Energy and Nuclear Physics of the U.S. Department of Energy under Contract DE-AC03-76SF00098.

\section{REFERENCES}

1) P. Bergè, Y. Pomeau and C. Vidal, "Order within Chaos," John Wiley \& Son, New York (1984).

2) D. Campbe11 in "Los Alamos Science," No.15, 1987, p.21a.

3) M.V. Berry, "Regular and Irregular Motion," in Topics in Nonlinear Dynamics (American Institute of Physics, New York, 1978), vol.46. p.16.

4) A.J. Lichtenberg and M.A. Lieberman, "Regular and Stochastic Motion," Springer-Verlag, New York, 1983.

5) E.J. Heller and P.W. O'Connor in proc. International Conference on the Physics of Chaos and Systems far from Equilibrium, H. Duong-Van, ed., North-Holldnd, 1987: Nuc 1. Phys. B (Proc. Supp 1.) 2 (1987) 201.

6) R. Arvieu, F. Brut and J. Carbonel1, Phys. Rev. A35 (1987) 2389;

]. Carbonel1, "Trajectoires classiques dans un potential nucléaire moyen," University of Grenoble thesis, 1983, preprint ISN 83-07.

7) V.M. Strutinsky, A.G. Hagner, S.R. Ofengenden and T. Odssing, Zeit. F. Phys. A283 (1977) 269.

8) R. Balian and C. Bloch, Ann. of Phys. $\underline{60}$ (1970) 401 .

9) 0. Bohigas and H.A. Wejdenmijller, "Aspects of Chaos in Nuclear Physics," Reprint HPI H-1988-V6, Hax-Planck-Institut für Kernphysik, Heidelberg, prepared for publication in Ann. Rev. Nuc 1. Part. Science.

10) 0. Bohigas, private communication, 1988.

11) W.J. Swiatecki, in "Semiclassical Descriptions of Atomic and Nuclear Collisions," North Holland, 1985, p.287.

12) O.L. Hill and J.A. Wheeler, Phys. Rev. Bg (1953) 1102.

13) G. Süssmann, Zeit. F, Physik, 139 (1954) 543. 
i4) F. Brut, private comunication, 1987.

15) C.F. Tsang, "On the Microscopic and Macroscopic Aspects of Nuclear Structure with Applications to Superheavy Nuclei," Ph.D. Thesis, University of California Lawrence Radiation Laboratory preprint UCRL-18899, May 22, 1969.

16) C. Yannouleas, Nuc 1. Phys. A439 (1985) 336; C. Yannouleas, M. Dworzecka and J.J. Griffin. Nucl. Phys. A339 (1980) 219.

17) W. Nörenberg, in "New Vistas in Nuclear Dynamics," ed. by P.J. Brussard and J.H. Koch, Plenum Press, New York, 1986.

18) K.T.R. Davies, K.R.S. Oevi, S.E. Koonin and M.R. Strayer, vol.3, p.3 in ref. 23.

19) W.U. Schröder and J.R. Huizenga, vol.2, p.115 in ref. 23.

20) W.Q. Shen et al., Phys. Rev. C36 (1987) 115.

21) j. Blocki, Y.-J. Shi and W.J. Swiateck1, "Order, Chaos and Nuclear Dynamics," in preparation.

22) J. Blocki et al., Ann. of Phys. 113 (1978) 330.

23) "Treatise on Heavy Ion Science," D.A. Bromley, ed., Volumes 1-7, Plenum, New York \& London, 19BA. 